\title{
Stercoral perforation of the colon proximal to an end colostomy
}

\author{
J.W. Serpell, M. Sen, G. Giddins' ${ }^{1}$ R.J. Nicholls and W.J.D. Bradfield ${ }^{1}$
}

Department of Surgery, St Thomas' Hospital, Lambeth Palace Road, London SE1 7EH and 'Department of Surgery, Kingston Hospital, Wolverton Avenue, Kingston upon Thames, Surrey KT2 7QB, UK

\begin{abstract}
Summary: Stercoral perforation of the colon is rare, and has not previously been reported as a postoperative complication, proximal to an end colostomy. Two such cases are reported; in addition in one the stercoral perforation was recurrent, emphasizing the multifocal nature of the disease. Both cases demonstrate the failure of standard techniques to deal with scybala in the loaded proximal colon. It is, therefore, suggested that intra-operative orthograde colonic lavage is indicated to protect a terminal colostomy from the risk of stercoral perforation in such cases.
\end{abstract}

\section{Introduction}

Despite Grinvalsky's ${ }^{1}$ finding of an overall incidence of $4.6 \%$ of stercoral ulceration at postmortem, stercoral perforation of the colon is a rarely reported condition. ${ }^{2}$ We report the first two cases of stercoral perforation occurring as a postoperative complication proximal to an end colostomy.

\section{Case reports}

\section{Case 1}

A 34 year old female presented with a 6 hour history of severe lower abdominal pain. On examination she had a temperature of $38^{\circ} \mathrm{C}$ and a tachycardia of 120 . There was tenderness and guarding in the lower abdomen, but rectal examination was normal. Vaginal examination revealed a tender mass in the right fornix and a presumptive diagnosis of a pyosalpinx was made. Appropriate antibiotic therapy was instituted; however, after failing to improve over a 12 hour period, laparoscopy was performed. This revealed a perforation of the sigmoid colon with faecal peritonitis. Subsequent laparotomy confirmed this to be a stercoral perforation of the lower sigmoid colon, with multiple perforations along a $6 \mathrm{~cm}$ segment of colon. This segment was excised, the rectal stump oversewn, peritoneal lavage performed, and an end colostomy created in the left iliac fossa after

Correspondence: J.W. Serpell, F.R.A.C.S., Alfred Hospital, Commercial Road, Prahran, Victoria 3181, Australia.

Accepted: 16 October 1990 manually emptying the loaded proximal colon of faecal content. The patient was discharged 7 days later after an uneventful recovery.

However, 24 hours later she returned with signs of generalized peritonitis. At laparotomy a recurrent stercoral perforation was present $6 \mathrm{~cm}$ proximal to the end colostomy. This segment was excised and the colostomy refashioned. Her subsequent course was uneventful and the colostomy was closed 3 months later. Pathological examination of both specimens revealed multiple stercoral perforations with ischaemic necrosis of the surrounding bowel wall. There was no intrinsic colonic pathology or vasculitis in the excised mesentery.

\section{Case 2}

A 53 year old male presented with a 9 month history of rectal bleeding, passage of mucus, tenesmus and gross constipation. On examination an extensive circumferential, stenosing, immobile carcinoma was present in the lower third of the rectum. Biopsy confirmed adenocarcinoma of the rectum. At laparotomy a massive, fixed tumour was found completely occupying the pelvis, and the proximal colon was loaded with scybala. A defunctioning left iliac fossa colostomy was created and a mucous fistula fashioned in the lower end of the wound to avoid obstruction in the defunctioned distal bowel. Five days postoperatively the colostomy was functioning satisfactorily. On the tenth day, however, the patient experienced colicky abdominal pain and distension, although the colostomy continued to function. Eighteen hours later generalized peritonitis was present, and plain abdominal X-rays revealed colonic distension, but 
no free gas. At laparotomy, the findings were of a diffuse faeculent peritonitis due to a stercoral perforation of the colon immediately proximal to the exit site of the colostomy. A limited sigmoid colectomy was performed, the colon manually milked of its contents and the colostomy refashioned. Four months later his condition was satisfactory. Histological examination confirmed multiple stercoral ulcers, with a full thickness perforating ulcer.

\section{Discussion}

Stercoral perforation has been reported proximal to an end transverse colostomy. ${ }^{3}$ However, the two cases described are the first reported cases to our knowledge occurring as a postoperative complication, proximal to an end sigmoid colostomy.

The diagnosis, as in the majority of previous reports, was not made prior to laparotomy. The first patient did not admit to constipation and radiological features of faecal loading were absent. However, there was a long history of marijuana abuse, which is a known constipative agent, ${ }^{4}$ and may have contributed to stercoral ulceration. Faecal impaction proximal to the stenosing tumour was recognized in the second case. However, despite careful manual emptying of the proximal colon at the initial laparotomy, this manoeuvre was not adequate to prevent stercoral perforation.

Both cases demonstrate the typical pathology of stercoral perforation, with multiple, round or ovoid, ischaemic ulcers, depressed below mucosa level, and a marked inflammatory reaction. ${ }^{1}$ These features distinguish the condition from idiopathi追 perforation where the primary pathology is a linear tear of the colonic wall, without associated inflam matory or ischaemic changes. ${ }^{2}$

The principles of treatment as outlined by? Guyton et al. ${ }^{5}$ were applicable to our cases, and involved preoperative resuscitation, elimination of all faecal soiling, resection of involved bowel with exteriorization and antibiotic therapy. Further more, the first case demonstrates the multifocalnature of this disease, which affects a segment rather than a focal point of the colon. This is the first reported case of recurrent stercoral perfora tion, and emphasizes the necessity fully to resect theo diseased segment, rather than exteriorization of the perforation alone.

Intra-operative orthograde colonic lavage is used to protect colonic anastomoses, and is effect-or ive at cleaning the colon of faecal content. ${ }^{6}$ The two cases described have demonstrated a failure of standard techniques to deal with the problem of the loaded proximal colon, even when an end colo- stomy is established. It is possible that stercora perforation might have been avoided in these twos cases had intra-operative orthograde colonic lavage been undertaken at the original operatiogs.

We therefore suggest that intra-operative orth -grade colonic lavage be performed to protecta terminal colostomy where the proximal colon is loaded with scybala, and is therefore at risk of stercoral perforation.

\section{References}

1. Grinvalsky, H.T. \& Bowerman, C.I. Stercoraceous ulcers of the colon; relatively neglected medical and surgical problem. JAMA 1956, 171: 1941-1946.

2. Beradi, R.S., Lee, S.S., Chen, H.P. \& Stines, G.J. Stercoraceous and spontaneous perforations of the colon. Int Surg 1987, 72: 235-240.

3. Gekas, P.\& Schuster, M.M. Stercoral perforation of the colon. Case report and review of the literature. Gastroenterology 1981, 80: 1054-1058.

4. Bowman, W.C. \& Rand, M.J. Textbook of Pharmacology, 2nd Ed., Blackwell Scientific Publications, Oxford, 1980, p. 52. 5. Guyton, D.P., Evans, D. \& Schreiber, H. Stercoral perforation of the colon. Concepts of operative management. Am Surg 1985, 51: $520-522$.

6. Koruth, N.M., Krukowski, Z.H., Youngson, G.G. et al: Intra-operative colonic irrigation in the management of left sided large bowel emergencies. Br J Surg 1985, 72: 708-711. 